\title{
Editorial: Biomedical Signals for Human-Computer Interaction
}

\author{
Hugo Plácido da Silva ${ }^{1 *}$, Nuno M. Garcia ${ }^{2}$ and Erin T. Solovey ${ }^{3}$ \\ ${ }^{1}$ Instituto de Telecomunicações (IT), Lisboa, Portugal, ${ }^{2}$ University of Beira Interior, Covilhã, Portugal, ${ }^{3}$ Worcester Polytechnic \\ Institute, Worcester, MA, United States
}

Keywords: biomedical engineering, human-computer interaction, biosignal acquisition, human factors, physiological computing

Editorial on the Research Topic

Biomedical Signals for Human-Computer Interaction

Over the past decades, the application of computer science and engineering principles to the field of Human-Computer Interaction (HCI) has proven to be of paramount importance, leading to remarkable technical, methodological and scientific achievements (Stephen, 2009). Biomedical signals are a particularly popular topic within the global research community. Recent examples spread through academia and industry, including initiatives such as the brain-computer interface (BCI) research program at Facebook Reality Labs (Tekla, 2020), the heart rate enhanced communication being explored by Snap, Inc. Research (Liu et al., 2021), or the work of Yamaha Corporation and world-renowned dancer Kaiji Moriyama on the use of Electromyography (EMG) to control a piano (Yamaha Corporation, 2018).

Nowadays, biomedical signals applied to HCI drive researchers in areas ranging from computer

OPEN ACCESS

Edited and reviewed by: Anton Nijholt, University of Twente, Netherlands

*Correspondence: Hugo Plácido da Silva hsilva@/x.it.pt

Specialty section:

This article was submitted to Human-Media Interaction,

a section of the journal Frontiers in Computer Science

Received: 22 October 2021 Accepted: 01 November 2021 Published: 18 November 2021

Citation:

Plácido da Silva H, Garcia NM and Solovey ET (2021) Editorial: Biomedical Signals for Human-

Computer Interaction.

Front. Comput. Sci. 3:799952. doi: 10.3389/fcomp.2021.799952 science and electrical engineering to social sciences, being one of the areas with high potential to enhance the way people leverage digital interaction. Although particularized to Interactive Music Systems (IMSs), the work by (Aly et al., 2021) presents a comprehensive review on the appropriation of biomedical signals as control structures. However, such interfaces still present several challenges in what concerns their design, creation and usefulness.

Many questions regarding the application of biosignals to next-generation HCI remain open: What information can be extracted from each modality and used for HCI? How can the systems be easy-to-use and a part of the user's daily life? How should the usefulness and effectiveness of interfaces be evaluated? What biosignal-mediated HCI approaches will result in practical and powerful interfaces for real-world deployment? How will the collaboration between researchers affect the architectural framework of the next generation of interfaces? This Research Topic presents outstanding work that answers multiple of these questions, describing the use of biomedical signals for next generation computer interfaces, and offering novel perspectives from theoretical and practical standpoints.

Westerink et al. explore the use of skin conductance measurements as a stress indicator, using cortisol as a benchmark. Cortisol is the main stress hormone in our body, and the study entitled "Deriving a cortisol-related stress indicator from wearable skin conductance measurements" provides a model for the relation between the skin conductance and the salivary cortisol response curves, along with the experimental evaluation of the method. The findings derived by the authors further reinforce the validity of skin conductance as a stress indicator.

User interface design and aesthetical evaluation is a classical area of interest regarding the use of biosignals for HCI. In this Research Topic, Ning et al. present three studies on the impact of spokescharacter faces in mobile application icons and brand evaluation. Entitled "The impact of incomplete 
faces of spokes-characters in mobile application icon designs on brand evaluations," finds that incomplete faces spokes-characters improve brand evaluation, and create perceptions of anthropomorphism.

Another area of interest in the interface between biosignal acquisition and HCI is gaming. In their work "Applications of biological and physiological signals in commercial video gaming and game research," Hughes and Jorda provide a review of the latest work in the use of biosignals for video game research and consumer applications. Particular emphasis is given to immersive experiences that incorporate biosignals as input and output channels in relation to the user. The relevance to gaming is further reinforced by the work of Dias et al., who present a case study on co-design of serious games to support Parkinson's disease management with the paper "Assistive HCI-serious games co-design insights: The case Study of i- PROGNOSIS personalized game suite for Parkinson's disease".

\section{REFERENCES}

Aly, L., Silvada Silva, H., Bernardes, G., and Penha, R. (2021). Appropriating Biosensors as Embodied Control Structures in Interactive Music Systems. Hum. Technology. 17 (1), 45-80. doi:10.17011/ht/urn.202106223978

Liu, F., Park, C., and Yu, J. (2021). "Significant Otter: Understanding the Role of Biosignals inCommunication," in Proc. of the ACM Conference on Human Factors in Computing Systems (CHI '21), Yokohama, Japan, May 8-13, 2021, 334-363.

Stephen, H. (2009). Fairclough. Fundamentals of Physiological Computing. Interacting Comput. 21 (Issues 1-2), 133-145. doi:10.1016/j.intcom.2008.10.011

Tekla, S. (2020). Perry. Here's How Facebook's Brain-Computer Interface Development Is Progressing. February: IEEE Spectrum.

Yamaha Corporation (2018). Yamaha Artificial Intelligence (AI) Transforms a Dancer into a Pianist. Available at: https://www.yamaha.com/en/news_release/ 2018/18013101/(Accessed August 13, 2021).
Overall the work published in this Research Topic further reinforces the maturity and growing relevance of biomedical signals in the design of modern HCI systems. Foreseen future work perspectives include the exploration of cultural and geographical factors in behavioral and emotion related research, further characterizing the relation of biomarkers such as cortisol with peripheral biomedical signals, harnessing the power of novel Artificial Intelligence (AI) tools to improve the quality of HCI experiences mediated by biomedical signals, and expanding the fields.

\section{AUTHOR CONTRIBUTIONS}

All authors listed have made a substantial, direct, and intellectual contribution to the work and approved it for publication.

Conflict of Interest: The authors declare that the research was conducted in the absence of any commercial or financial relationships that could be construed as a potential conflict of interest.

Publisher's Note: All claims expressed in this article are solely those of the authors and do not necessarily represent those of their affiliated organizations, or those of the publisher, the editors and the reviewers. Any product that may be evaluated in this article, or claim that may be made by its manufacturer, is not guaranteed or endorsed by the publisher.

Copyright (C) 2021 Plácido da Silva, Garcia and Solovey. This is an open-access article distributed under the terms of the Creative Commons Attribution License (CC BY). The use, distribution or reproduction in other forums is permitted, provided the original author(s) and the copyright owner(s) are credited and that the original publication in this journal is cited, in accordance with accepted academic practice. No use, distribution or reproduction is permitted which does not comply with these terms. 